\title{
Comparación del perfil emprendedor en estudiantes del área empresarial e industrial México-Perú
}

Comparison of the entrepreneurial profile in students of the Mexico-Peru business and industrial area

Comparação do perfil empreendedor em estudantes da área comercial e industrial do México-Peru

María de Lourdes Amador Martínez Instituto Tecnológico Superior del Oriente del Estado de Hidalgo, México lamador@itesa.edu.mx https://orcid.org/0000-0001-8963-6264

Yessica García Hernández Instituto Tecnológico Superior del Oriente del Estado de Hidalgo, México ygarcia@itesa.edu.mx https://orcid.org/0000-0002-4482-7275

Jeanette Fabiola Díaz Quintanilla Universidad Católica San Pablo, Perú jfdiaz@ucsp.edu.pe https://orcid.org/0000-0002-1345-2810 


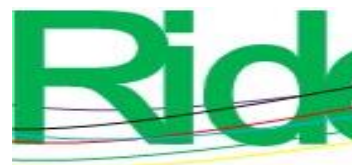

Revista Iberoamericana para la

Investigación y el Desarrollo Educativo

ISSN $2007-7467$

\section{Resumen}

Existen diversas estrategias diseñadas con la intención de fomentar el desarrollo y crecimiento económico en los países; una de ellas es el emprendimiento, principalmente entre los jóvenes. Por lo tanto, resulta fundamental el papel que desarrollan las instituciones de educación superior, especialmente las relacionadas de forma directa con el área empresarial e industrial. En la presente investigación se aborda dicho tema desde la teoría sistémica del emprendimiento, para lo cual se han considerado dos factores muy ligados entre sí: la cultura y la educación. El objetivo fue evaluar el perfil emprendedor de los estudiantes de educación superior del área empresarial e industrial de dos universidades (una mexicana y otra peruana) mediante el instrumento GUESSS (Global University Entrepreneurial Spirit Student's Survey). Como hipótesis se planteó que existe diferencia significativa entre el perfil emprendedor de los estudiantes de los dos países. Para ello, se desarrolló una investigación cuantitativa con alcance descriptivo. La recolección de datos se llevó a cabo mediante formularios de Google, los cuales se enviaron por correo electrónico a los alumnos seleccionados (276 mexicanos y 323 peruanos). Las respuestas conseguidas se pasaron a Excel y posteriormente a SPSS (versión 24) para realizar su análisis descriptivo. Los resultados indican que el perfil emprendedor de los estudiantes en México es de 4.32 y en Perú de 3.87. Esta diferencia resulta significativa entre los grupos mediante la prueba t de Student obteniendo $\mathrm{f}=0.000$, por lo que se comprueba la hipótesis de que existe diferencia significativa.

Palabras clave: intención emprendedora, GUESSS, estudiantes universitarios.

\section{Abstract}

There are various strategies designed with the intention of promoting development and economic growth in the countries; one of them is entrepreneurship, mainly among young people. Therefore, the role played by higher education institutions is essential, especially those directly related to the business and industrial area. In the present investigation, this topic is approached from the systemic theory of entrepreneurship, for which two factors closely linked to each other have been considered: culture and education. The objective was to evaluate the entrepreneurial profile of higher education students from the business and industrial area of two universities (one Mexican and one Peruvian) through the GUESSS instrument (Global University Entrepreneurial Spirit Student's Survey). As a hypothesis, it was proposed that there is a significant difference between 


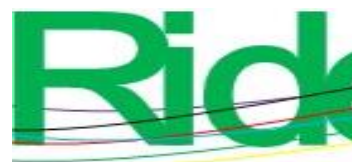

Revista Iberoamericana para la Investigación y el Desarrollo Educativo

ISSN $2007-7467$

the entrepreneurial profile of the students of the two countries. For this, a quantitative research with a descriptive scope was developed. Data collection was carried out using Google forms, which were sent by email to the selected students (276 Mexicans and 323 Peruvians). The answers obtained were transferred to Excel and later to SPSS (version 24) to carry out their descriptive analysis. The results indicate that the entrepreneurial profile of students in Mexico is 4.32 and in Peru 3.87. This difference is significant between the groups using the Student's t-test, obtaining $\mathrm{f}=0.000$, so the hypothesis that there is a significant difference is confirmed.

Keywords: Entrepreneurial intention, GUESSS, university students.

\section{Resumo}

Existem várias estratégias elaboradas com a intenção de promover o desenvolvimento econômico e o crescimento nos países; Um deles é o empreendedorismo, principalmente entre os jovens. Portanto, é essencial o papel das instituições de ensino superior, especialmente aquelas diretamente relacionadas à área comercial e industrial. Esta pesquisa trata dessa questão a partir da teoria sistêmica do empreendedorismo, para a qual foram considerados dois fatores intimamente ligados: cultura e educação. O objetivo foi avaliar o perfil empreendedor de estudantes do ensino superior da área comercial e industrial de duas universidades (uma mexicana e uma peruana), utilizando o instrumento GUESSS (Pesquisa Global de Estudantes de Espírito Empresarial da Universidade). Como hipótese, afirmou-se que há uma diferença significativa entre o perfil empreendedor dos estudantes dos dois países. Para isso, foi desenvolvida uma investigação quantitativa com escopo descritivo. A coleta de dados foi realizada por meio de formulários do Google, que foram enviados por e-mail aos alunos selecionados (276 mexicanos e 323 peruanos). As respostas obtidas foram repassadas ao Excel e posteriormente ao SPSS (versão 24) para realizar sua análise descritiva. Os resultados indicam que o perfil empreendedor dos estudantes no México é 4,32 e no Peru 3,87. Essa diferença é significativa entre os grupos que utilizam o teste t de Student, obtendo $\mathrm{f}=0,000$, sendo verificada a hipótese de que há uma diferença significativa.

Palavras-chave: intenção empreendedora, GUESSS, estudantes universitários.

Fecha Recepción: Agosto 2019

Fecha Aceptación: Enero 2020 


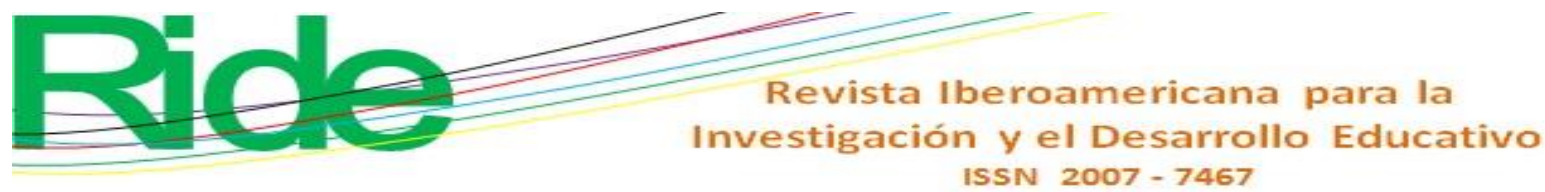

\section{Introducción}

Desde hace algunos años, el emprendimiento ha ocupado diversos espacios en la academia, en la investigación y en las políticas públicas, pues este tema constituye uno de los factores indispensables para consolidar la pirámide empresarial de los países debido a los aportes que genera en el ámbito de creación y desarrollo de nuevas oportunidades (Secretaría de Economía, 2017).

Dada la importancia del tema, en el plan nacional de México 2013-2018 —específicamente en la meta México próspero- se ha procurado ofrecer las mismas oportunidades a los emprendedores con el fin de erradicar paulatinamente los programas asistenciales y con ello superar los niveles de pobreza. En este sentido, el grupo de atención primordial está constituido por los jóvenes, principalmente aquellos que se encuentran en su preparación universitaria.

En concordancia con este propósito, Perú se unió en el año 2003 al proyecto Global Entrepreneurship Monitor (GEM). Según reporte del GEM 2016/17, esta nación ocupa el cuarto lugar en cuanto al emprendimiento en América Latina, pues 25 de cada 100 peruanos se encuentran involucrados en algún tipo de actividad emprendedora. El GEM clasifica a los países (más específicamente, a sus economías) de acuerdo con la fase de desarrollo económico en que se encuentran según las categorías usadas en el Reporte Global de Competitividad del Foro Económico Mundial (Schwab y Sala-I-Martin, 2012). Esta categorización se sustenta en el producto interno bruto per capita y en la proporción de bienes primarios en las exportaciones, entre otros indicadores. Sus categorías son las siguientes: economías basadas en recursos, economías basadas en eficiencia y economías basadas en innovación.

Según Díaz (2010), en los últimos diez años en Perú ha habido un incremento de la actividad emprendedora debido principalmente al crecimiento económico. Esto ha generado el incremento del poder adquisitivo, así como una mayor capacidad de ahorro y de consumo entre los jóvenes que demandan más productos y servicios innovadores y con mayor valor agregado. Asimismo, se percibe una más alta valoración hacia los emprendedores como personas con valentía para enfrentar nuevos retos. Esto, sumado a la existencia de algunos programas destinados a la generación del autoempleo, ha producido una revaloración del empresario.

Muchos autores coinciden en que conocer el ecosistema del emprendimiento es importante porque permite determinar sus componentes, el aprendizaje de las mejores prácticas, la búsqueda de nuevas oportunidades y los modelos para facilitar el camino a los nuevos emprendedores (Hernández y González, 2016). 


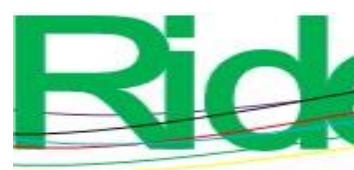

Revista Iberoamericana para la Investigación y el Desarrollo Educativo

ISSN $2007-7467$

Por todo lo anterior, las instituciones de educación superior (IES) deben preocuparse cada vez más por formar egresados que cuenten con las competencias necesarias para ser competitivos. El objetivo es fomentar una mentalidad empresarial en los estudiantes, sobre todo en aquellos cuyo perfil se vincula con la creación de empresas propias.

Señalado lo anterior, se puede indicar que la importancia de la presente investigación se encuentra en que se intenta ofrecer un diagnóstico claro sobre las diferencias existentes en cuanto al perfil emprendedor de estudiantes del área empresarial e industrial de México y Perú, pues de esa manera se pueden promover acciones que gradualmente robustezcan la iniciativa emprendedora.

\section{Revisión de literatura}

La formación enfocada en la creación de empresas es un objetivo estratégico para elevar los niveles de innovación, competitividad, crecimiento económico y generación de empleo de un país, de ahí que en los jóvenes universitarios se deban desarrollar sus aptitudes y actitudes. Para lograr tal fin, sin embargo, antes se deben identificar sus potencialidades y debilidades para que las estrategias de orientación aplicadas se ajusten a sus realidades.

Históricamente, el emprendimiento se ha considerado como una fórmula para generar empleo y para lograr un modelo de desarrollo socioeconómico alternativo, el cual incorpora y mejora el bienestar social de aquellos individuos excluidos del mercado laboral formal a pesar de que cuenten con una carrera universitaria (Rangel, Alvarado y Sierra, 2016). Por ello, este tema ha resultado de suma importancia para las universidades, la comunidad y las empresas, pues se busca crear escenarios de participación que sean capaces de ofrecer alternativas productivas y el fomento de una cultura emprendedora (Fuentes y Sánchez, 2010).

Ahora bien, en cuanto al término emprendedor, Orrego (2008) señala que se deriva del vocablo latino prendere, que significa 'acometer e intentar', de ahí que haya sido usado en principio para referirse a los pioneros que decidían correr riesgos o lanzarse a una aventura. Por su parte, Gutiérrez y Amador (2011) refieren que el emprendedor universitario es aquella persona con estudios superiores que tiene el conocimiento suficiente y está motivado para iniciar y desarrollar sus sueños de una manera innovadora, asertiva y proactiva, llevándolo a producir impactos en la rama productiva elegida. 


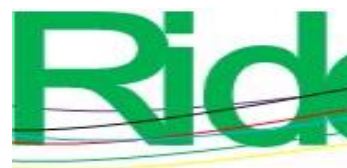

Revista Iberoamericana para la Investigación y el Desarrollo Educativo

ISSN $2007-7467$

El emprendimiento, por su parte, se puede definir como la capacidad de una persona para identificar oportunidades en su entorno, con la finalidad de alcanzar beneficios e impactar en la sociedad, para lo cual debe correr ciertos riesgos financieros (Sepúlveda et al., 2017). El emprendimiento, lógicamente, exige el descubrimiento, la evaluación y la explotación de oportunidades para introducir nuevos bienes y servicios, formas de organización, y procesos y materiales (Delgado, Gómez, Romero, y Vázquez, 2008).

Aunado a lo anterior, la expresión iniciativa emprendedora o espíritu emprendedor se asocia a la necesidad de establecer el perfil emprendedor en los individuos. Al respecto, Lerma (2007) lo define como un perfil que muestra con claridad la serie de características, habilidades y potenciales que, como educadores o detentadores del poder, se pueden aprovechar como marco de referencia para identificar, incentivar y cultivar el capital humano emprendedor. En 1937, Ely y Hess (citados por Chan, Escalante y Robles, 2015) conceptualizaron el perfil emprendedor como un conjunto de características que permiten actuar de una manera determinada y mostrar ciertas "capacidades" para visualizar, definir y alcanzar objetivos.

Para Guerrero (2016) potenciar la actividad emprendedora en las IES requiere planificación previa y conocimiento de dónde se está en cada momento, identificando con esto el punto de partida, la fase intermedia y la etapa final, siempre y cuando se quieran lograr resultados útiles; por lo tanto, un correcto diagnóstico es imperante para dirigir acciones concretas. En concordancia con esta idea, Fernández, Rueda y Herrero (2011) recomiendan que las IES ofrezcan a los estudiantes cursos que mejoren sus conocimientos empresariales, los cuales se deben poner en práctica en situaciones reales.

Por otra parte, vale agregar que diversas investigaciones consideran que la acción emprendedora tiene un fuerte vínculo con distintos factores psicológicos, contextuales y sociodemográficos (Espí, Arana, Heras y Díaz de Junguitu, 2007). Uno de los modelos de intención que más repercusión ha tenido es la teoría del comportamiento planeado de Ajzen (1991), que se refiere la facilidad o dificultad de las personas para alcanzar el logro que buscan mediante su conducta. Esta teoría se centra en las intenciones de elección de carrera en general y en las intenciones emprendedoras en particular.

La intención de un individuo suele estar influenciada por la actitud personal hacia los resultados del comportamiento, es decir, hacia las consecuencias probables de su acción; en segundo lugar, abarca la presión percibida de las normas subjetivas que subyacen en el entorno y, 


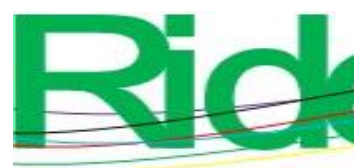

Revista Iberoamericana para la Investigación y el Desarrollo Educativo

ISSN $2007-7467$

por último, comprende el control percibido de su futuro comportamiento (Ajzen, 1991). Esto significa que la creación de un nuevo negocio o empresa requiere necesariamente de una planificación, por lo que representa el tipo de comportamiento planeado ideal para estudiar a través de un modelo de intención.

Está teoría está constituida por cuatro dimensiones: actitud personal hacia el emprendimiento, control del comportamiento percibido, intención emprendedora y normas subjetivas, las cuales, en consecuencia, definen la iniciativa de un estudiante hacia el emprendimiento.

En el caso específico de México, sin embargo, autores como Kantis (2004) señalan que existe una disminución del capital humano emprendedor, de la cultura emprendedora, así como de la percepción de las políticas referentes al tema. Por su parte, Hernández, Sánchez, Díaz y Fernández (2017) afirman que las instituciones educativas en México, de manera tradicional, no han formado a los estudiantes con habilidades ni capacidades de emprendimiento; por ello, los estudiantes al egresar no cuentan con una mentalidad emprendedora, de ahí que se conformen con laborar en una empresa establecida o en la administración pública. Pero esta situación se puede revertir tomando en cuenta lo referido por Guerrero (2016), quien explica que se debe promover un espíritu emprendedor entre los universitarios. Por ello, Niebles-Núñez, Hernández-Palma y Cardona-Arbeláez (2016) consideran importante que los representantes de las instituciones educativas comprendan que la gestión del conocimiento tiene impactos positivos en la creación de desarrollo.

Asimismo, el GEM (2014) recomienda que la política pública de México contemple dos elementos: el capital financiero para la conformación de mipymes y el capital social para el emprendimiento; en este sentido, existe una oportunidad en la educación para generar competencias emprendedoras y de los negocios, principalmente, en temas de resistencia, resiliencia e identificación de oportunidades, así como la generación de ideas innovadoras buscando tolerancia.

En el caso de Perú, la sugerencia se enfoca en el marco de promoción del crecimiento, reducción de la pobreza y reforzamiento de las leyes con el fin de mejorar su posición de emprendimiento. Al respecto, Mitchelmore y Rowley (2010) indican que los programas elaborados deben procurar que el estudiante se enfrente a experiencias críticas de desarrollo, pues de ese modo 


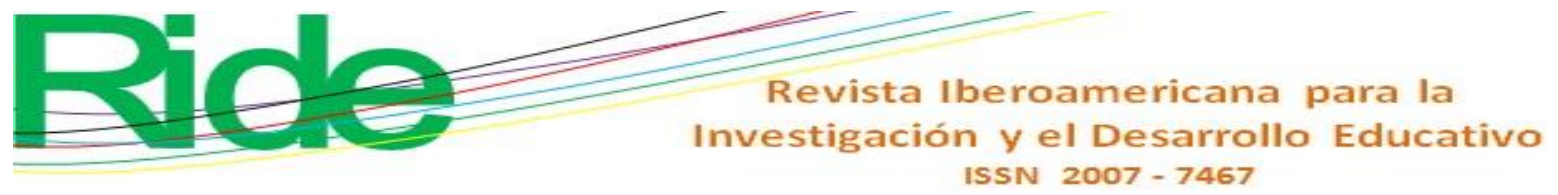

se pueden consolidar los conocimientos y las habilidades adquiridas, lo que redundará en resultados positivos.

Hoy en día en distintas latitudes se han realizado diversas investigaciones respecto a la iniciativa emprendedora en los estudiantes. En España, por ejemplo, se pueden mencionar los trabajos de Espí et al. (2007), quienes hallaron que perfil emprendedor de estudiantes de la Universidad del País Vasco es de tipo medio-bajo, mientras que en una indagación efectuada en otra universidad pública de España, Fuentes y Sánchez (2010) demostraron que existe una menor iniciativa emprendedora en las mujeres. Igualmente, el proyecto GUESSS (Global University Entrepreneurial Spirit Student's Survey) llevado a cabo en la Universidad de Granada muestra que los estudiantes de ingeniería y arquitectura presentan mayor vocación emprendedora que los de las áreas de ciencias sociales y humanidades (Fuentes et al., 2015).

En cuanto a las investigaciones desarrolladas en México, Espíritu (2011) se enfocó en determinar la influencia que ejercen los tres rasgos de personalidad (locus de control interno, necesidad de logro y propensión al riesgo) sobre la intención emprendedora de 333 estudiantes de las carreras de Contador Público y licenciado en Administración de la Universidad de Colima. Los resultados revelan una alta relación positiva significativa entre los tres rasgos de personalidad y la intención de crear sus propias empresas.

En ese mismo año, Gutiérrez y Amador (2011) realizaron una investigación para comparar el potencial emprendedor de los estudiantes de Contabilidad en un centro universitario de Perú y en uno de Guadalajara (México). Los hallazgos demuestran que la iniciativa para emprender en ambas universidades se situó en un punto medio, mientras que las competencias con mejor manejo en los alumnos de las dos escuelas fueron la fortaleza ante las dificultades, el aprendizaje y el afán de logro. En cambio, las que alcanzaron mayor deficiencia fueron la capacidad de asumir riesgos, la capacidad de tomar decisiones, la flexibilidad, la organización de tiempo y trabajo y la confianza en sí mismos.

Espíritu, González y Alcaraz (2012) analizaron el grado de competencias emprendedoras de 315 estudiantes de las áreas Contable y Administración, así como el rol que juegan los docentes con el fin de conocer si están incentivando la actitud emprendedora entre sus alumnos. Los resultados evidencian que existe una mayor probabilidad de la intención emprendedora en los estudiantes de la licenciatura en Administración. 


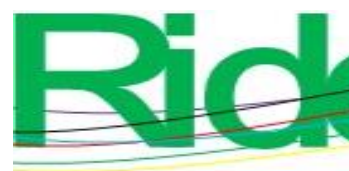

De Jorge (2013), por su parte, analizó los factores que promueven la intención emprendedora de los estudiantes universitarios; sus resultados muestran que las características sociodemográficas en términos de mayor edad, género masculino y existencia de algún familiar empresario son causas explicativas de la intención emprendedora. Este autor, además, explica que para concretar un determinado comportamiento de emprendedor se requieren una actitud positiva hacia ese comportamiento, la norma subjetiva o apoyo percibido del entorno más próximo al individuo y el control del comportamiento percibido.

Ibars, Corona, Trujillo, Torreblanca y Zazueta (2018), en cambio, se centraron en analizar si la motivación de origen causal percibido interno tiene incidencia en la permanencia en el proceso de incubación empresarial de los emprendedores en el Instituto Tecnológico de Veracruz (ITV). Los resultados muestran que existe una correlación baja entre la motivación interna de los emprendedores hacia la permanencia en el proceso de incubación; además, los emprendedores que terminaron el proceso de incubación tienden a tener mayor pasión que compromiso personal por sus proyectos.

En cuanto a los antecedentes peruanos, cabe destacar el trabajo de Pérez (2009), quien exhorta a la universidad para que contribuya a la formación de los estudiantes en materia del emprendimiento empresarial, en especial en lo referente a desarrollo de competencias, habilidades y destrezas que permitan una mayor generación y aprovechamiento de las oportunidades que brinda el mercado. Este enfoque requiere del compromiso de las autoridades universitarias, responsables de establecer los lineamentos que orientan la visión y misión de los centros de formación superior.

Por último, Zorrilla (2013) se enfoca en identificar las competencias orientadas al mercado y la actitud emprendedora que fueron aplicadas en el proyecto Jóvenes Pilas, el cual ha servido para que los emprendedores participantes tuvieran mejoras en su persona, negocio y entorno; por tanto, los hallazgos suman aportes a la política de Estado para el emprendimiento juvenil y a las mypes.

En definitiva, se debe enfatizar que las instituciones educativas se tienen que interesar por la formación del espíritu emprendedor de los jóvenes mediante la incorporación de asignaturas con un enfoque de formación integral que abarque diversas áreas del conocimiento y que permita a los estudiantes consolidar este tipo de habilidades (Solís, Alhuay, Hernández, Lingan y Rodríguez, 2017). 


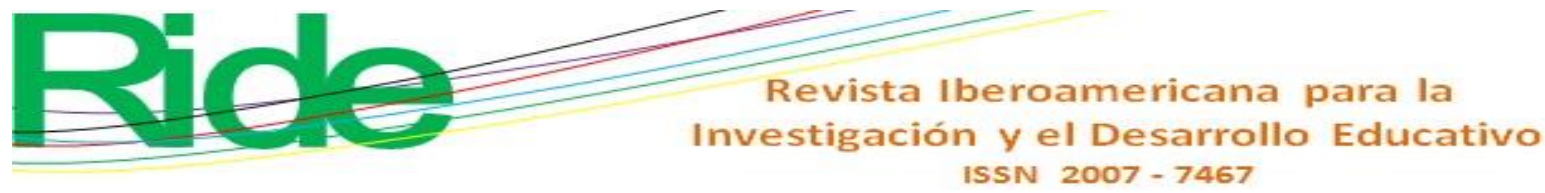

\section{Planteamiento del problema}

De acuerdo con el Plan Nacional de Desarrollo 2013-2018, la desocupación en México se concentra principalmente en los jóvenes, pues $53 \%$ de personas desocupadas tienen entre 14 y 29 años de edad, con una tasa de ocupación de $7.9 \%$. Estas cifras demuestran un problema en cuento a la inclusión de este grupo de personas al mercado laboral, aunque también abre la posibilidad de crear nuevas oportunidades que promuevan la actitud emprendedora. Sin embargo, cabe indicar que en la práctica resultan escasas las iniciativas de este tipo (Kantis, 2014), de ahí que sea fundamental analizar el papel de las instituciones sobre dicho tema.

Por ello, sobresale el Modelo Talento Emprendedor empleado en el Tecnológico Nacional de México, el cual pretende fomentar la creación de empresas por parte de los futuros profesionistas. No obstante, aún hace falta un diagnóstico específico para conocer con certeza cuál es la intención emprendedora de los estudiantes y especialmente para identificar los factores que deben ser desarrollados con el fin de mejorar la ejecución del referido modelo; aunado a esto, no se cuenta con un estudio que aborde el tema del emprendimiento desde un enfoque predictivo, como sucedería con la teoría del comportamiento planificado.

En Perú, según Falconi —expositor del Programa de Emprendimiento iLab de la Universidad de Piura - son dos los factores que dificultan la tarea de emprender: el primero son las personas, ya que se ha demostrado una relación directa entre la confianza y el emprendimiento; en otras palabras, el éxito del emprendimiento se consigue solo cuando se encuentran personas confiables y comprometidas que sepan trabajar en equipo. El segundo factor es el proceso de constitución de las empresas y la falta de incentivos, de ahí que se deban brindar capacitaciones en instituciones educativas que ofrezcan herramientas a los emprendedores para superar esta falencia (América Economía, 2015).

Por eso, el Gobierno peruano viene desarrollando programas de emprendimiento que buscan generar negocios que puedan ser financiados. Asimismo, las universidades han procurado potenciar el espíritu emprendedor de jóvenes; una muestra de ello es la Universidad Católica San Pablo, en cuyas instalaciones se llevó a cabo la capacitación de emprendimiento de negocios a cargo de especialistas de Corea y el programa GET (global entrepreneurship training), el cual ha sido avalado por la Unesco. 


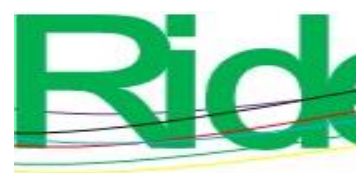

Revista Iberoamericana para la Investigación y el Desarrollo Educativo

ISSN $2007-7467$

Gracias a este tipo de iniciativas, según Serida, Alzamora, Guerrero, Borda y Morales (2016), Perú se ha venido convirtiendo en uno de los países más emprendedores de Latinoamérica, lo que puede traducirse en que existen grandes oportunidades de crecimiento para los emprendimientos. Parte de las oportunidades de crecimiento mencionadas se hacen visibles en el tercer trimestre del año 2017.

Explicado lo anterior, se puede decir que la pregunta que ha orientado la presente investigación ha sido la siguiente: ¿cuál es el nivel del perfil emprendedor de los estudiantes del área empresarial e industrial de dos instituciones de educación superior de México y Perú?

\section{Justificación}

Esta investigación procura contribuir a la mejora gradual del perfil emprendedor de los estudiantes de dos IES de México y Perú, pues se ha demostrado que la universidad es uno de los espacios más importantes en cuanto a la generación del capital humano y social para los emprendedores (González y Velázquez, 2015). Sobre este aspecto, resulta significativo resaltar que los estudiantes requieren una sólida formación en materia de emprendimiento para poder gestionar sus propias empresas (Duval-Couetil, Gotch y Yin, 2014).

Asimismo, vale resaltar que el enfoque usado (es decir, la teoría del comportamiento planificado) puede servir para predecir lo que sucederá y para establecer estrategias que permitan fortalecer el Modelo Talento Emprendedor, a partir de un diagnóstico que determine el impacto que tienen los siguientes factores: institución, programa educativo, género, semestre, antecedentes de emprendimiento, formación en materia de emprendimiento, tradición emprendedora, actitud personal hacia el emprendimiento, normas subjetivas, control de comportamiento percibido e iniciativa emprendedora en la intención emprendedora (Espíritu, 2011; Medina, Bolívar y Lemes, 2014).

\section{Objetivo general}

Evaluar el perfil emprendedor de los estudiantes del área empresarial e industrial de dos Instituciones de Educación Superior, mediante el instrumento GUESSS, con la finalidad realizar una comparación entre México y Perú. 


\section{Hipótesis}

De acuerdo con la evaluación del perfil emprendedor en estudiantes de educación superior, se procede a desarrollar la siguiente hipótesis:

- Existe diferencia significativa en el perfil emprendedor de los estudiantes del área empresarial de México y Perú.

\section{Metodología}

La presente investigación se sustentó en un enfoque cuantitativo, con un corte transversal, exploratorio y descriptivo. La variable dependiente fue el perfil emprendedor, mientras que la independiente fue el país de origen de los estudiantes (México o Perú). Los datos fueron analizados mediante el software estadístico SPSS (versión 25).

\section{Desarrollo del estudio (discusión-análisis)}

Para el desarrollo de la investigación, en primera instancia, se determinó la muestra con que se trabajaría según cada una de las IES, como se indica a continuación.

\section{Muestra}

Al tratarse de un estudio comparativo, para llevar a cabo el cálculo de la muestra se utilizó la fórmula para poblaciones finitas. Para el caso de México se tomó el total de las carreras de ingeniería en Gestión Empresarial, licenciatura en Administración e ingeniería en Logística. De acuerdo con la matrícula del semestre enero-junio 2018 (fecha de aplicación del instrumento), había un total de 970 estudiantes distribuidos en los diferentes semestres. Conocida esta cifra, se procedió a usar la siguiente fórmula:

$$
n=\frac{(z) 2 p * q * N}{(N)(e) 2+(z) 2 p * q}
$$




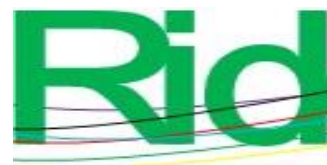

Donde

$$
\begin{gathered}
(1.96) 2(0.50) *(0.50) *(941) \\
n=(970)(0.05) 2+(1.96) 2(0.50) *(0.50) \\
=276
\end{gathered}
$$

En el caso de Perú, se consideró a la población total de la carrera Ingeniería Industrial, por lo que se tomó a los diez semestres. La población estuvo constituida por 2048 alumnos, de modo que la muestra fue determinada como se muestra a continuación:

$$
n=\frac{(z) 2 p * q * N}{(N)(e) 2+(z) 2 p * q}
$$

Donde

$$
\begin{gathered}
(1.96) 2(0.50) *(0.50) *(941) \\
n=(2048)(0.05) 2+(1.96) 2(0.50) *(0.50) \\
=323
\end{gathered}
$$

En síntesis, la aplicación se hizo a 276 estudiantes de México y a 323 de Perú.

\section{Instrumento de medición}

El instrumento usado fue una adaptación del GUESSS (Global University Entrepreneurial Spirit Student's Survey), el cual tiene como propósito comprender la intención y la actividad emprendedora de los estudiantes universitarios (Medina et al., 2014). Este consta de tres apartados: el primero se utilizó para conocer las variables sociodemográficas (institución, programa educativo, género y semestre). El segundo estuvo constituido por preguntas de respuestas cerradas que permitieron recabar información sobre los antecedentes en materia de emprendimiento, la tradición emprendedora y la formación en materia de emprendimiento, así como la intención de elección de la carrera universitaria con enfoque hacia el pasado y futuro. Por último, el tercero fue elaborado con 18 ítems centrados en cuatro factores del perfil emprendedor: intención emprendedora, actitud personal hacia el emprendimiento, control del comportamiento percibido y normas subjetivas. La escala usada fue de tipo Likert ( $1=$ muy en desacuerdo; 2 = en desacuerdo; 


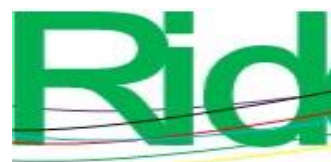

Revista Iberoamericana para la Investigación y el Desarrollo Educativo

ISSN $2007-7467$

$3=$ neutral; 4 = de acuerdo; 5 = muy de acuerdo). El instrumento fue validado en una prueba piloto, en la cual se obtuvo un alfa de Cronbach de 0.912 .

\section{Recolección de información}

La recolección de datos se llevó a cabo mediante formularios de Google, los cuales se enviaron por correo electrónico a los alumnos seleccionados. Las respuestas conseguidas se pasaron a Excel y posteriormente a SPSS (versión 24) para realizar su análisis descriptivo. De este modo se contrastó la hipótesis con la prueba t de Student para muestras independientes.

\section{Resultados}

Los resultados del presente trabajo indican que del total de encuestados, $46 \%$ estudian en la universidad de México y $54 \%$ en la universidad de Perú. Asimismo, 37.3\% son hombres y $62.7 \%$ mujeres.

En cuanto a la intención de emprender, en la tabla 1 se evidencia que en los tres momentos analizados (intención de elegir carrera, finalización de carrera y cinco años después haberla culminado) predomina el propósito Ser fundador y trabajar en su propia empresa, mientras que en menor porcentaje se ubica la opción Ser sucesor en una empresa familiar; esta última cifra demuestra que la mayoría de los estudiantes no tiene familiares dedicados a los negocios empresariales. 


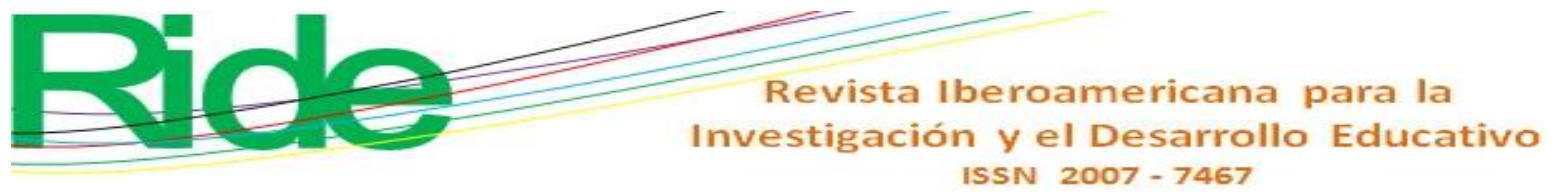

Tabla 1. Intención de elección de carrera

\begin{tabular}{|l|c|c|c|}
\hline \multicolumn{1}{|c|}{ Descripción } & $\begin{array}{c}\text { ¿Cuál fue la } \\
\text { intención al elegir } \\
\text { la carrera? }\end{array}$ & $\begin{array}{c}\text { ¿Cuál es su intención } \\
\text { al terminar la carrera? }\end{array}$ & $\begin{array}{c}\text { ¿Cuál es su intención 5 } \\
\text { años después de } \\
\text { terminarla? }\end{array}$ \\
\hline $\begin{array}{l}\text { Trabajar en una empresa } \\
\text { privada al terminar la } \\
\text { carrera. }\end{array}$ & 28.8 & 36.3 & 16.5 \\
\hline $\begin{array}{l}\text { Trabajar en una empresa } \\
\text { pública al terminar la carrera }\end{array}$ & 10.5 & 10.5 & 5.7 \\
\hline $\begin{array}{l}\text { Ser fundador y trabajar en su } \\
\text { propia empresa }\end{array}$ & 51.8 & 45.0 & 70.0 \\
\hline $\begin{array}{l}\text { Ser sucesor en empresa } \\
\text { familiar }\end{array}$ & 3.3 & 2.8 & 2.3 \\
\hline No lo sé todavía. & 5.5 & 5.3 & 5.5 \\
\hline Total & 100.0 & 100.0 & 100.0 \\
\hline
\end{tabular}

Fuente: Elaboración propia

A partir de lo anterior, se presentan los resultados de la hipótesis central del presente trabajo:

- H1: Existe diferencia significativa en el perfil emprendedor de los estudiantes del área empresarial entre México y Perú.

En relación con la hipótesis planteada, en la tabla 2 y en la figura 1 se presentan los resultados de la comparación de medias en primera instancia para muestras independientes; al respecto, se consideró la evaluación del perfil emprendedor constituida por cuatro dimensiones: la iniciativa emprendedora, el control de comportamiento percibido, las normas subjetivas y la actitud personal hacia el emprendimiento. Estas, cuando se analizan de forma integral, generan el perfil emprendedor de acuerdo con el instrumento de GUESSS aplicado en los dos países.

Las cifras indican que la diferencia de medias en México fue de 4.32, mientras que en Perú fue de 3.87. Por ello, se puede decir que el perfil emprendedor es más elevado en el caso de México.

Tabla 2. Comparación de medias del perfil emprendedor (México y Perú)

\begin{tabular}{|c|c|c|c|c|}
\hline País & $\mathrm{N}$ & Media & $\begin{array}{l}\text { Desviación } \\
\text { estándar }\end{array}$ & $\begin{array}{l}\text { Error } \\
\text { estándar }\end{array}$ \\
\hline México & 276 & 4.32 & 0.960 & 0.058 \\
\hline Perú & 323 & 3.87 & 1.310 & 0.073 \\
\hline
\end{tabular}

Fuente: Elaboración propia 
Figura 1. Puntuaciones medias del perfil emprendedor

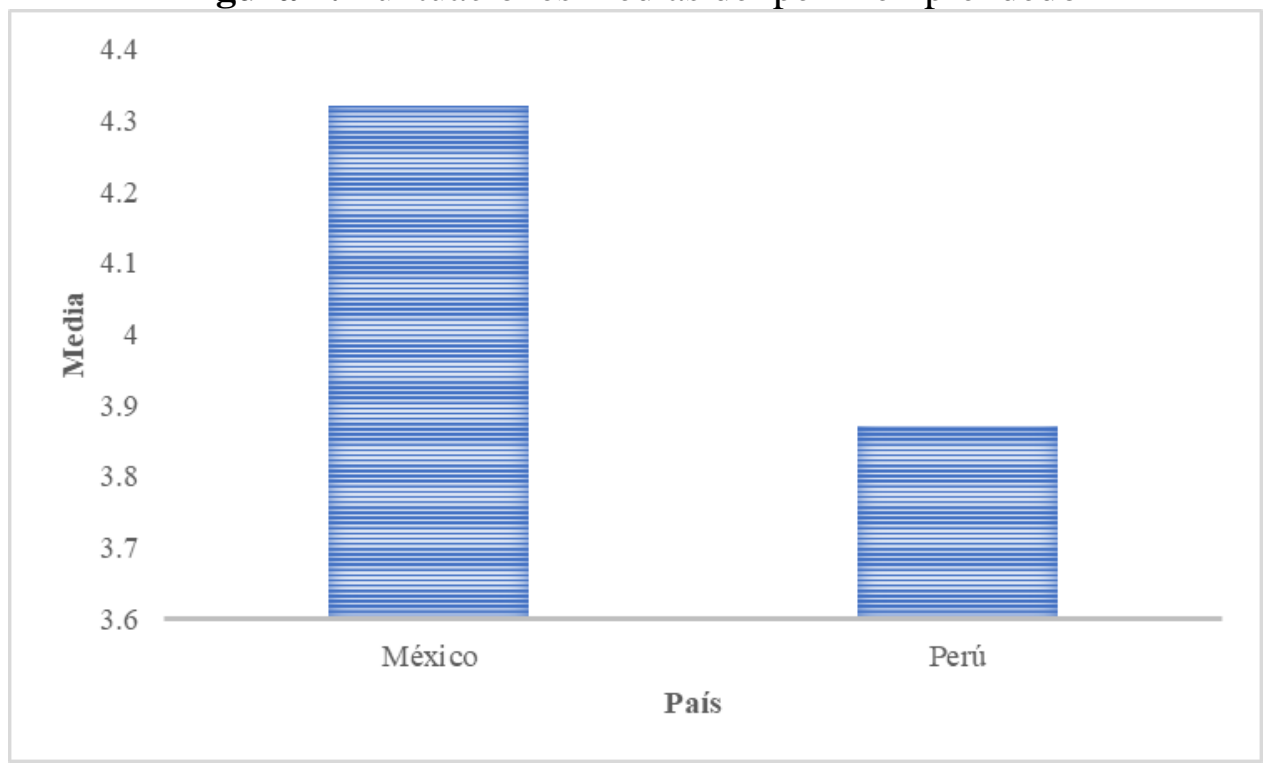

Fuente: Elaboración propia

Ahora bien, como ya se indicó, se empleó la prueba t de Student para muestras independientes, donde en primera instancia se considera que se está trabajando con varianzas iguales debido al valor de significancia de .000 , ya que el valor es menor a .005 . Para poder contrastar la hipótesis se tomaron los datos de t y se siguió la regla de decisión, es decir, si el nivel de significancia calculado es menor o igual a 0.05 , se debe aceptar la hipótesis. Por lo tanto, se considera que existe diferencia significativa en el perfil emprendedor de los estudiantes del área empresarial entre México y Perú.

Tabla 3. Prueba t de Student para comparar el perfil emprendedor México y Perú

\begin{tabular}{|c|c|c|c|c|c|}
\hline $\begin{array}{c}\text { Perfil } \\
\text { emprendedor }\end{array}$ & F & Sig. & t & $\begin{array}{c}\text { Significancia } \\
\text { bilateral }\end{array}$ \\
\hline & $\begin{array}{c}\text { Se asumen varianzas } \\
\text { iguales }\end{array}$ & 51.377 & .000 & 4.674 & 0.000 \\
\hline & $\begin{array}{c}\text { No se asumen varianzas } \\
\text { iguales }\end{array}$ & & & 4.786 & 0.000 \\
\hline
\end{tabular}

Fuente: Elaboración propia 


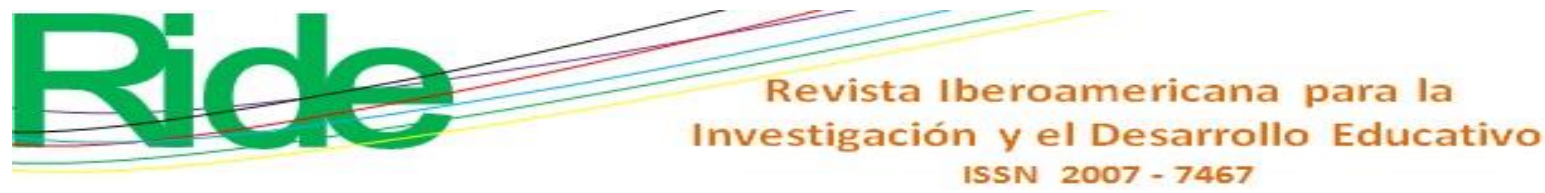

\section{Discusión}

La presente investigación se realizó en dos momentos y con el apoyo del Cuerpo Académico de Gestión Empresarial (para el caso de México) y de la maestra Jeanette Fabiola Díaz Quintanilla (para el caso de Perú). Asimismo, vale acotar que existe la posibilidad de que el estudio realizado pueda ser complementado con entrevistas en estudios posteriores. De hecho, se puede especificar el perfil emprendedor de los estudiantes según el género y de acuerdo con el grado de avance en la carrera que cursan. Además, se podría concretar una indagación longitudinal para comparar los datos obtenidos, considerando las políticas públicas implementadas en los dos países. De hecho, al ser estas dos naciones latinoamericanas con muchas similitudes, existe la posibilidad de generar propuestas que se puedan desarrollar para mejorar sus niveles de emprendimiento.

\section{Conclusiones}

Actualmente, la dinámica económica demanda el fomento del emprendimiento como una opción para fortalecer el desarrollo de los países y para convertirse en una opción laboral para los futuros profesionistas. Por ello, resulta fundamental el papel que asuman las IES como formadoras de emprendedores, especialmente aquellas que ofertan programas educativos del área empresarial e industrial.

Con la presente investigación se ha logrado el objetivo de analizar el perfil emprendedor de los estudiantes del área empresarial e industrial de dos universidades (una de México y otra de Perú). En tal sentido, se puede afirmar que en dichas instituciones los estudiantes (no solo al iniciar y finalizar la carrera, sino incluso cinco años después de haberla culminado) tienen la intención de ser fundadores y trabajar en su propia empresa. Esto significa que la formación profesional ofrecida en las IES analizadas desarrolla el espíritu emprendedor de los alumnos, lo cual se debe seguir consolidando para que esa intención logre materializarse de la mejor manera. En otras palabras, se puede pensar en la apertura o la actualización constante de las asignaturas relacionadas con el emprendimiento. Como señalan Asai, Flores, Montiel, Saavedra y Tapia (2015), una misión de las IES actuales es promover el emprendimiento y la generación de empresas de un alto valor agregado que sean capaces de innovar para la prosperidad. 


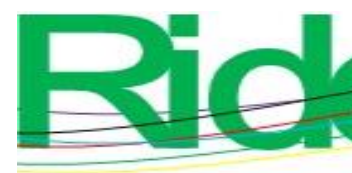

Revista Iberoamericana para la

Investigación y el Desarrollo Educativo

ISSN $2007-7467$

Por otra parte, se ha demostrado que las mujeres constituyen el mayor número de estudiantes en las carreras analizadas y que existe un nivel de desarrollo superior en México que en Perú, lo cual ofrece la oportunidad de profundizar en las razones de estas diferencias. Aunado a esto, se debe considerar que en el presente estudio solo se tomaron en cuenta dos universidades, por lo que se debería ampliar la muestra para tener una visión más amplia de este tema.

En general, se puede concluir que aspectos como la limitada oferta de trabajo y las dificultades asociadas a la búsqueda del primer empleo han generado una presión favorable para estimular el espíritu emprendedor de los estudiantes.

\section{Referencias}

Ajzen, I. (1991). The theory of planned behavior. Organizational behavior and human decision processes, 50(2), 179-211.

América Economía (2015). Estos son los principales problemas de los peruanos al emprender. Recuperado de https://mba.americaeconomia.com/articulos/notas/estos-son-losprincipales-problemas-de-los-peruanos-al-emprender

Asai, Y., Flores, M. Y., Montiel, M., Saavedra, M. L. y Tapia, B. (2015). El caso de la Universidad Nacional Autónoma de México. En Emprendimiento femenino en Iberoamérica (pp.109134). México: RedEmprendia.

Chan, M. R., Escalante, N. S. y Robles, E. (2015). Comparabilidad en el nivel de emprendimiento de los alumnos de dos universidades: México y Puerto Rico. Revista Internacional Administración y Finanzas, 8(5), 1-14.

De Jorge, J. (2013). Análisis de los factores que influyen en la intención emprendedora de los estudiantes universitarios. Caracciolos, 1(1), 1-12.

Delgado, P. M. I., Gómez, M. L., Romero, M. A. M., y Vázquez, I. E. (2008). Determinantes sociales y cognitivos en el espíritu emprendedor: un estudio exploratorio entre estudiantes argentinos. Cuadernos de Gestión, 8(1), 11-2.

Díaz, E. (2010). Fortaleciendo el ecosistema de los startups en el Perú a Través de La Ruta del Startup Peruano. Recuperado de https://www.centrumthink.pucp.edu.pe/Docs/files/fortaleciendo_el_ecosistema_de_los_st 


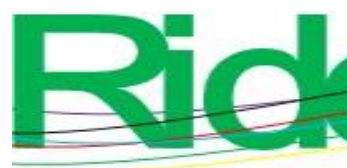

Revista Iberoamericana para la

Investigación y el Desarrollo Educativo

ISSN $2007-7467$

artups_a_trav\%C3\%A9s_de_la_ruta_del_startup_peruano_-_d\%C3\%ADaz_-

_hopkins_(1).pdf.

Duval-Couetil, N., Gotch, C. and Yin, S. (2014). The characteristics and motivations of contemporary entrepreneurship students. Journal of Education for Business, 89, 441-449.

Espí, M. T., Arana, G., Heras, I. y Díaz de Junguitu, A. (2007). Perfil emprendedor del alumnado universitario del campus de Gipuzkoa de la UPV/EHU. Revista de Dirección y Administración de Empresas, (14), 83- 110.

Espíritu, R. (2011). Análisis de la intención emprendedora en estudiantes universitarios a través de los rasgos de personalidad. Multiciencias, 11(1), 65-75.

Espíritu, R., González, R. F. y Alcaraz, E. (2012). Desarrollo de competencias emprendedoras: un análisis explicativo con estudiantes universitarios. Cuadernos de Estudios Empresariales, 22, 29-53.

Fernández, A., Rueda, M. I. y Herrero, A. (2011). Estudio de la actitud emprendedora de los estudiantes universitarios de la facultad de CC. EE. y empresariales de Cantabria. XVI Congreso de la Asociación Española de Contabilidad y Administración de Empresas (AECA). 1-22.

Fuentes, F. J. y Sánchez, S. M. (2010). Análisis del perfil emprendedor: una perspectiva de género. Estudios de Economía Aplicada, 28(3), 1-28.

Fuentes, M. M., Pérez, F., Ruiz, M., Albacete, C. A., Bojica, A. M. y Ruiz, J. M. (2015). Espíritu emprendedor de los estudiantes de la Universidad de Granada. España: Universidad de Granada.

Global Entrepreneurship Monitor [GEM] (2014). Informe GEM España 2011. Global Entrepreneurship Research Association.

González, M. D. y Velázquez, L. M. (2015). El caso del Tecnológico de Monterrey. En Emprendimiento femenino en Iberoamérica (pp. 135-154). México: RedEmprendia.

Guerrero, M. (2016). Perfil emprendedor del estudiante universitario. Madrid: Red Emprendida.

Gutiérrez, A. M. y Amador, M. E. (2011). El potencial emprendedor en los estudiantes de la carrera de Contabilidad de las universidades San Marcos de Perú y Guadalajara de México - Centro Universitario de los Altos - un análisis comparativo. Revista de la Facultad de Ciencias Contables, 19(36), 63-82. 


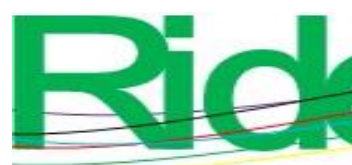

Revista Iberoamericana para la Investigación y el Desarrollo Educativo

ISSN $2007-7467$

Hernández, C. y González, D. (2016). Study of the Start-Up Ecosystem in Lima, Peru: Collective Case Study. Latin American Business Review, 17(2), 115-137.

Hernández, R., Sánchez, M. C., Díaz, J. C. y Fernández, A. (2017). Educación emprendedora universitaria. Enfoque de micro ecosistemas. Conferência sobre Educação para o Empreendedorismo, 223-257.

Ibars, S. M., Corona, E. E., Trujillo, P. C., Torreblanca, A. y Zazueta, B. E. (2018). Motivación interna de los emprendedores en el proceso de incubación empresarial. Revista Global de Negocios, 6(1), 45-54.

Kantis, H. (ed.) (2004). Desarrollo emprendedor. América Latina y la experiencia internacional. Washington, EE. UU.: Banco Interamericano de Desarrollo.

Lerma, A. E. (2007). Mida su estatura como emprendedor. Organización y Dirección, 59-63.

Medina, P., Bolívar, A. y Lemes, A. I. (2014). Un paso más en la investigación de la intención emprendedora del estudiante universitario: GUEESS. Revista de Estudios Empresariales, 2, 63-80.

Mitchelmore, S. and Rowley, J. (2010). Entrepreneurial Competencies: A Literature Review and Development Agenda. International Journal of Entrepreneurial Behaviour \& Research, 16(2), 92-111.

Niebles-Núñez, W., Hernández-Palma, H., y Cardona-Arbeláez, D. (2016). Gestión tecnológica del conocimiento: herramienta moderna para la gerencia de instituciones educativas. Revista de Investigación, Desarrollo e Innovación, 7(1), 25-36.

Orrego, C. I. (2008). La dimensión humana del emprendimiento. Revista Ciencias Estratégicas, 16(20), 225-235.

Pérez, E. (2009). La universidad en la formación de emprendedores empresariales y el apoyo en la creación de nuevas empresas. Gestión en el Tercer Milenio, 12(23).

Rangel, L. O., Alvarado, M. M. y Sierra, O. E. (2016). El perfil emprendedor como catalizador de la innovación en los jóvenes del Departamento de la Guajira. Omnia, 22(3), 71-86.

Schwab, K. and Sala-I-Martin, X. (2012). Insight Report: The Global Competitiveness Report 2012-2013. Ginebra: Foro Económico Mundial.

Secretaría de Economía (2017). Emprendedores. Recuperado de http://www.20062012.economia.gob.mx/mexico-emprende/empresas/emprendedor. 


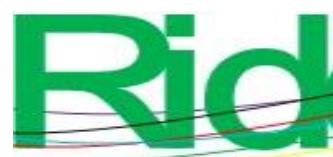

Revista Iberoamericana para la Investigación y el Desarrollo Educativo

ISSN $2007-7467$

Sepúlveda, M. J., Denegri, C. M., Orellana, C. L., Criado, N., Mendoza, J., Salazar, P. y Yung, G. (2017). Características emprendedoras personales y alfabetización económica: una comparación entre estudiantes universitarios del sur de Chile. Interdisciplinaria, 34(1), 107-124.

Serida, J., Alzamora, J., Guerrero, C., Borda, A. y Morales, O. (2016). Global Entrepreneurship Monitor: Perú 2015-2016. Lima: ESAN. Recuperado de http://repositorio.esan.edu.pe/handle/ESAN/635.

Solís, C., Alhuay, J., Hernández, R., Lingan, S. y Rodríguez, J. (2017). Evaluación y desarrollo del potencial emprendedor en estudiantes universitarios de Lima, Perú. Recuperado de http://repositorio.usil.edu.pe/bitstream/USIL/2625/1/2017_Solis_Evaluacion-desarrollopotencial-emprendedor-en\%20estudiantes-universitarios-Lima-Peru.pdf.

Zorrilla, D. (2013). Factores que contribuyeron a la sostenibilidad de los micro emprendimientos juveniles en el distrito de San Juan de Lurigancho. Caso: Proyecto Jóvenes Pilas del Programa de Empleo Juvenil de INPET (2007-2009) (tesis de maestría). Pontificia Universidad Católica del Perú. Recuperado de http://tesis.pucp.edu.pe/repositorio/bitstream/handle/123456789/5508/zorrilla_mejia_davd _factores_inpet.pdf?sequence $=1$. 
Revista Iberoamericana para la Investigación y el Desarrollo Educativo ISSN 2007 - 7467

\begin{tabular}{|c|c|}
\hline Rol de Contribución & Autor (es) \\
\hline Conceptualización & (María de Lourdes, (principal) Yessica, (igual) Jeanette (que apoya)) \\
\hline Metodología & (Yessica, (principal) María de Lourdes (que apoya)) \\
\hline Software & (María de Lourdes, Yessica, Jeanette (igual)) \\
\hline Validación & (María de Lourdes, Yessica, Jeanette (igual)) \\
\hline Análisis Formal & (María de Lourdes) \\
\hline Investigación & (María de Lourdes y Jeanette (igual)) \\
\hline Recursos & (Yessica, María de Lourdes y Jeanette (igual)) \\
\hline Curación de datos & (María de Lourdes y Jeanette (igual)) \\
\hline $\begin{array}{l}\text { Escritura - Preparación del borrador } \\
\text { original }\end{array}$ & (María de Lourdes, Jeanette y Yessica (igual)) \\
\hline Escritura - Revisión y edición & María de Lourdes) \\
\hline Visualización & (María de Lourdes) \\
\hline Supervisión & (María de Lourdes y Yessica (igual)) \\
\hline Administración de Proyectos & (María de Lourdes) \\
\hline Adquisición de fondos & (Yessica, María de Lourdes (igual) y Jeanette (apoya)) \\
\hline
\end{tabular}

